income countries (LMIC). The objective is to develop a tool that (1) identifies the essential components of an urban emergency response system, (2) outlines methods for identifying gaps among components, (3) recommends best practices for addressing these gaps, and (4) recommends improvement strategies to policy makers.

Methods A systematic review of literature was conducted to identify existing tools, scoring systems and urban emergency response indices. Then, in-depth interviews (IDIs), and focus group discussions (FGDs) were held with key stakeholders to identify essential response components. This was followed by a modified Delphi process with 25 emergency response experts, to score each component on a scale of 1 to 4 ( 1 being least critical and 4 being most critical). The highest ranked components were included in the City Emergency-health Response Capability (CERC) tool.

Results The literature review yielded 20 articles defining components to an emergency response system. Then 25 IDIs and12 FGDs independently identified further components, which were subsequently ranked in the Delphi process. The highest ranked components were categorized into 5 domains: service delivery, safety and security, human resource, command and control, policy and procedures. The final CERC tool will use a set of scored questions and observations about these components in implementation.

Conclusion CERC aims to objectively identify gaps in LMIC urban emergency response systems. Using this tool, city officials can identify areas for improvement and resource allocation to increase their disaster-readiness

\section{P1.002 PREHOSPITAL TRAUMA BURDEN MANAGED BY A SOUTH AFRICAN PROVINCIAL EMERGENCY MEDICAL SERVICE}

${ }^{1}$ Naseef Abdullah, ${ }^{2}$ Michael McCaul, ${ }^{2}$ Peter Nyasulu, ${ }^{1}$ Colleen Saunders*. ${ }^{1}$ Division of Emergency Medicine, University of Cape Town, Observatory, South Africa; ${ }^{2}$ Division of Epidemiology and Biostatistics, Stellenbosch University, Tygerberg, South Africa

10.1136/injuryprev-2021-safety. 29

Background Trauma is one of the leading causes of death and disability in South Africa. There is a paucity of data describing the prehospital trauma burden and the aim of this study was therefore to describe the epidemiology of trauma emergencies managed by the Western Cape government Emergency Medical Service (WCG EMS) in South Africa.

Methods This retrospective study included a descriptive analysis of all trauma patients managed between July 2017 and June 2018.

Results The WCG EMS managed 492303 cases during the study period. of these cases, 168980 (34.3\%) were trauma cases. The majority of patients $(66.4 \%)$ were males and between the socio-economically active ages of 21-40 years old $(54.0 \%)$. Assaults were the most common cause of trauma emergencies, accounting for $50.2 \%$ of the EMS case load managed. The patient acuity was categorised as urgent for $47.5 \%$ of the cases, and $74.9 \%$ of the prehospital trauma burden was transported to a secondary level health care facility for definitive care.

Conclusion This is the first report of the prehospital trauma burden managed in the Western Cape of South Africa. The
Western Cape suffers a unique trauma burden with a high proportion of assault and violence related trauma

\section{P1.003 ASSESSMENT OF URBAN EMERGENCY RESPONSE SYSTEMS: A LITERATURE REVIEW}

Agnes Usoro*, Junaid Razzak. Johns Hopkins University, Baltimore, USA

10.1136/injuryprev-2021-safety.30

Background Emergency response and preparedness is a global priority, particularly in an urbanizing world. Urban emergency response systems require complex coordination between many stakeholders. However, there are no validated tools that evaluate a city's overall emergency response capacity and preparedness for disasters. To inform the development of such a tool, we conducted a literature review on the components, scoring systems, and the reliability of evaluation methods to measure urban emergency response and preparedness.

Methods A systematic search of PubMed, Google Scholar and EMBASE identified studies, guidelines, measures, and instruments focused on evaluating emergency response and preparedness at the hospital, city, and national levels between 1983 to 2017. Studies focusing on computer simulations, and those without measurement or scoring systems were excluded.

Results Out of 473 nonduplicate citations, 20 studies were included after full-text review describing 1) quantitative scoring methodologies, 2) key components of a response/preparedness plan, and 3) the development of assessment tools. Quantitative assessments of emergency preparedness or response used index scores, summary scores, performance scales, and observational checklists. Key readiness components included staff training, security and safety, leadership, and incident command structure; whereas staff knowledge, vulnerability, and functional recovery were critical for emergency response planning.

Conclusion A comprehensive emergency response and preparedness tool can be developed from clearly defined components, using quantitative methods such as indices or checklists. Such a tool could help in the assessment of a city's emergency preparedness and response capacity in a reproducible and objective manner, but will require validation in an urban environment.

\section{P1.004 IMPACTS OF SAFE COMMUNITY PROGRAMS ON SOCIAL LOSS IN JAPAN}

${ }^{1}$ Hiasto Imai* ${ }^{2}$ Yoko Shiraishi* ${ }^{1}$ Mature Life Insitute, Osaka City, Japan; ${ }^{2}$ Japan Institution for Safe Communities, Osaka City, Japan

10.1136/injuryprev-2021-safety.31

Background Since 2005 when the Safe Community programs (SC) based on the seven indicators was firstly introduced in Japan, there are 17 communities which have introduced the SC into their community development policies. Among those communities, there is growing interest in what impacts SC has made in their communities.

Methods The social cost of main causes of injuries such as suicide, traffic incidents, falls of the elderly was calculated 
based on the data provided by the fire department (ambulance transportation) and the medical institutions in some communities working on SC.

Results Although there are limitations to have the exact cost of the social loss under the different conditions in each community, in the case of community $\mathrm{A}$, one of the communities with longest experience of SC programs, we found that the community can save 18,081,333 USD (per 100,000) when they have zero suicide and depression.

Conclusion In this study, possible losses made from influence on their families and workplaces are not included to the calculation. With the consideration of those conditions, the social loss can be much higher. To see precise losses, in addition to direct losses, we should identify what make indirect influences on the social losses. and it can be different by different causes of injuries.

Learning Outcomes With the scandalized frame for the calculation of the social loss, we can show the benefits of prevention programs. It can convince policy makers to understand how much benefit the programs for injury prevention can make in their communities

\section{P1.005 ACTION PLAN FOR SAFETY, BASED ON LOCAL CONDITIONS, NATIONAL POLICIES, GLOBAL VISIONS}

Eva Vaagland*. Norwegian Safety Forum, Oslo, Norway

10.1136/injuryprev-2021-safety.32

Context Norwegian laws and regulations state that municipalities shall promote health and well-being, contribute to good environmental conditions and prevent mental and somatic illnesses and injuries. Preventive efforts are given broad attention.

This gives Norway a supportive framework for prevention, and a potential interest for safety work on community level. But public health work mainly focuses on lifestyle diseases and the prevention of these. Less effort is put into injury prevention and safety promotion. The Norwegian Safety Forum (NSF) is a non-profit organization, a national advocate for safety supported by the ministry of health. It's a a hub for information with activities, networking and meetings on the agenda. We want to engage local communities in safety promotion.

Process NSF has developed a national program for community safety based on local conditions, challenges and available resources, Norwegian laws and regulations, WHO visions, and Safe Community experiences. The program is developed to meet national targets and will guide municipalities to reach these goals by developing multisector collaboration and use documented prevention measures. NSF offers advice, guidance, tools, networks and seminars.

Outcome We have established a national network With about 40 municipalities using the program for community safety. The program is endorced by the National Program for Public Health, The National Program for Safe Elderly and the National Plan for Road Safety. The model with a step-by-step approach is a useful instrument for communities, turning global visions and national policies into local realities.

\section{A - Child - Road, March 23, 2021}

\section{A.001 ROAD TRAFFIC INJURIES AMONG CHILDREN AND ADULTS IN THE REPUBLIC OF MOLDOVA}

Angela Cazacu-Stratu, Svetlana Cociu*. Department of Hygiene 'Nicolae Testemitanu' State University of Medicine and Pharmacy, Republic of Moldova, Chisinau, Moldova (the Republic of)

\subsection{6/injuryprev-2021-safety.33}

Background Road accidents are a major problem for public health and a major cause of deaths and injuries worldwide. In the Republic of Moldova, road accidents are one of the mortality cause among children aged between 14-18 years old and one of the main causes of morbidity of children aged between $0-14$ years old. The aim of this study was to underline the particularities of road injuries and to develop effective preventive measures of reducing road injures.

Methods A retrospective study was performed with data collection from the official statistical reports of the Chisinau Police Department for 2010-2018, SPSS was used for data analysis.

Results Road injuries account for $1.2 \%$ of the total number of child injuries in the country and $1.4 \%$ in Chisinau. Mortality from road trauma in the country during the last 8 years registered the highest level in September (13.5\%) and June (11\%) and the fewest deaths were detected in March - 3.4\%, while in Chisinau, most deaths were registered in January, August and November (19.1\% each). In $46.2 \%$ of children were as a pedestrian. Men aged 20-29 years old and 50-59 years old were identified at higher risk of dying being a driver or being a passenger in a transport unit.

Conclusion Data showed a downward trend of $30.3 \%$, although it is necessary to strengthen the cross-sectoral actions and take common efforts in road safety.

Learning Outcomes Our results suggested the need for health promotion and health education campaigns and intersectoral approach among the vulnerable groups.

\section{A.002 CHILD SAFEGUARDING - IT'S A JOURNEY NOT A DESTINATION \\ ${ }^{1,2}$ Alan Foster* . 'City of Casey, Australia; ${ }^{2}$ Narre Warren, Australia}

10.1136/injuryprev-2021-safety.34

Background/Aims The City of Casey wanted to be aspirational and not just compliant when implementing the Victorian Child Safe Standards and Reportable Conduct Scheme.

Methods Council set out to embed a child safe culture throughout the organisation that would:

- demonstrate a zero-tolerance approach towards child abuse,

- ensure children and young people who engage with City of Casey services are safe and feel empowered to seek support,

- equip staff and volunteers to recognise the signs of child abuse and manage reportable incidents effectively and in compliance with legislation; and

- develop and maintain a robust governance framework. 